\title{
Development of dual-band antenna mounted on shoes for GPS and wide area network
}

\author{
Tetsuya Nakamura ${ }^{\text {a) }}$ and Yoshinobu Okano ${ }^{\text {b) }}$ \\ Graduate School of Engineering, Tokyo City University, \\ 1-28-1 Tamazutsumi, Setagaya-ku, Tokyo 158-8557, Japan \\ a) g1681527@tcu.ac.jp \\ b)y-okano@tcu.ac.jp
}

Abstract: Recently, the increase in aged wanderers or the infant abduction is becoming the serious social problem. The practical use of the GPS function loadings shoes is advanced to prevent such incident occurring. The shoes mountable dual-band antenna that can correspond to the GPS signal reception, and the uplink into the wide area network is proposed in this report. The GPS-band of the proposal antenna is used to acquire the positional information on shoes. On the other hand, acquired shoes positional information is uplinked to the wide area network by Wi-Fi-band, and is used to inform the police or the nurse.

Keywords: wearable antenna, dual-band antenna, GPS, WAN, UHF band Classification: Antennas and Propagation

\section{References}

[1] D. Gaetano, P. McEvoy, M. J. Ammann, J. E. Browne, L. Keating, and F. Horgan, "Footwear antennas for body area telemetry," IEEE Trans. Antennas Propag., vol. 61, no. 10, pp. 4908-4916, 2013. DOI:10.1109/TAP.2013. 2272451

[2] Y. Ono and Y. Okano, "Development of wearable antenna for VHF-band," Intelligent Signal Processing and Communications Systems, 2013. DOI:10. 1109/ISPACS.2013.6704624

[3] Y. Nakazato and Y. Okano, "A study of using re-radiation wearable antenna for digital terrestrial broadcasting," Asia-Pacific Microwave Conference, 2010.

[4] “The Report of National Institute of Bioscience and Human-Technology," 1994, in Japanese.

[5] http://www.fcc.gov/general/body-tissue-dielectric-parameters

[6] A. Taflove and M. E. Brodwin, "Numerical solution of steady state electromagnetic scattering problem using the time dependent Maxwell's equation," IEEE Trans. Microw. Theory Techn., vol. 23, no 8, pp. 623-630, 1975. DOI:10.1109/TMTT.1975.1128640 


\section{Introduction}

Recently, the increase in aged wanderers or the infant abduction is becoming the serious social problem. Especially, the development of a preventive technology of such an incident is strongly hoped for in the East Asian region where low birth rate and longevity progress rapidly. The technology that continuously observes elderlies or child's positions is practicable as a preventive technique of such an incident. The practical use of the GPS function loadings shoes is advanced to continuously observe the target person's position [1]. The antenna element is often stored in sole though the radiation efficiency decreases by user's foot in the GPS function loadings shoes. Moreover, the antenna that transmits measured positional information to the smart phone's network is also equipped into the sole. However, the development of the dual-band antenna and the investigation of antenna loading position become important problems in achievement of the antenna properties and shoe's comfortableness. Therefore, the development of the novel dual-band antenna (corresponding to GPS and Wi-Fi band) that can be loaded on the tiptoe of shoes is described in this report. The GPS-band of the proposal antenna is used to acquire the positional information on shoes. On the other hand, acquired shoes positional information is uplinked to the wide area network by Wi-Fi-band, and is used to inform the police or the nurse.

\section{Antenna structure}

Specifically the dual-band antenna corresponding to GPS band $(1.575 \mathrm{GHz})$ and Wi-Fi or Bluetooth band $(2.4 \mathrm{GHz})$ is proposed. In addition, it is assumed to load the dual-band antenna for shoes that will always be put on when the person goes out. The configuration of the proposal antenna and Positional relationship of the antenna and the foot model are shown in Fig. 1. The optimum antenna dimensions in free space are $H 1=13 \mathrm{~mm}, H 2=7 \mathrm{~mm}, K=2 \mathrm{~mm}, L 1=26 \mathrm{~mm}, L 2=17 \mathrm{~mm}$, $M=2 \mathrm{~mm}, D=20 \mathrm{~mm}$. The distance between the foot model and the dielectric spacer is $2 \mathrm{~mm}$. This is because the thickness of the shoe's skin was assumed to be about $2 \mathrm{~mm}$. Also the ground and the dielectric spacer are in contact. A basic concept of the proposal antenna derives from inverted-F antenna and T-shape monopole antenna. The shorting point and the feed point are set up around the center of main antenna element. The right hand side element as viewed from the feed point contributes to GPS-band and other side element contributes to Wi-Fiband. Moreover, a parasitic element is loaded above of the main antenna element for the impedance matching within Wi-Fi-band [2]. The antenna element is sandwiched between the dielectric spacer (relative permittivity $\varepsilon_{\mathrm{r}}=2.0$, conductivity $\sigma=0.01 \mathrm{~S} / \mathrm{m}$ ) to increase the durability. Because the wearable antenna is used near the human body, it is necessary to simulate the body effect for the antenna design [3]. Concretely, because the proposal antenna will be loaded in the shoe's tiptoe, it is necessary to simulate foot influence. The foot model's size is based on the Report of National Institute of Bioscience and Human-Technology [4]. The foot model is divided into muscle part (relative permittivity $\varepsilon_{\mathrm{r}}=36.5$, conductivity $\sigma=0.85 \mathrm{~S} / \mathrm{m}$ ) and bone part (relative permittivity $\varepsilon_{\mathrm{r}}=15.0$, conductivity $\sigma=$ $0.58 \mathrm{~S} / \mathrm{m})$. The electric constant of the foot model is drawn from the body tissue 
dielectric parameters database of the Federal Communications Commission [5]. In addition, it is necessary to consider the influence of the ground into the antenna properties. The asphalt is assumed as a stuff of the ground (relative permittivity $\varepsilon_{\mathrm{r}}=2.7$, conductivity $\left.\sigma=0.1 \mathrm{~S} / \mathrm{m}\right)$.
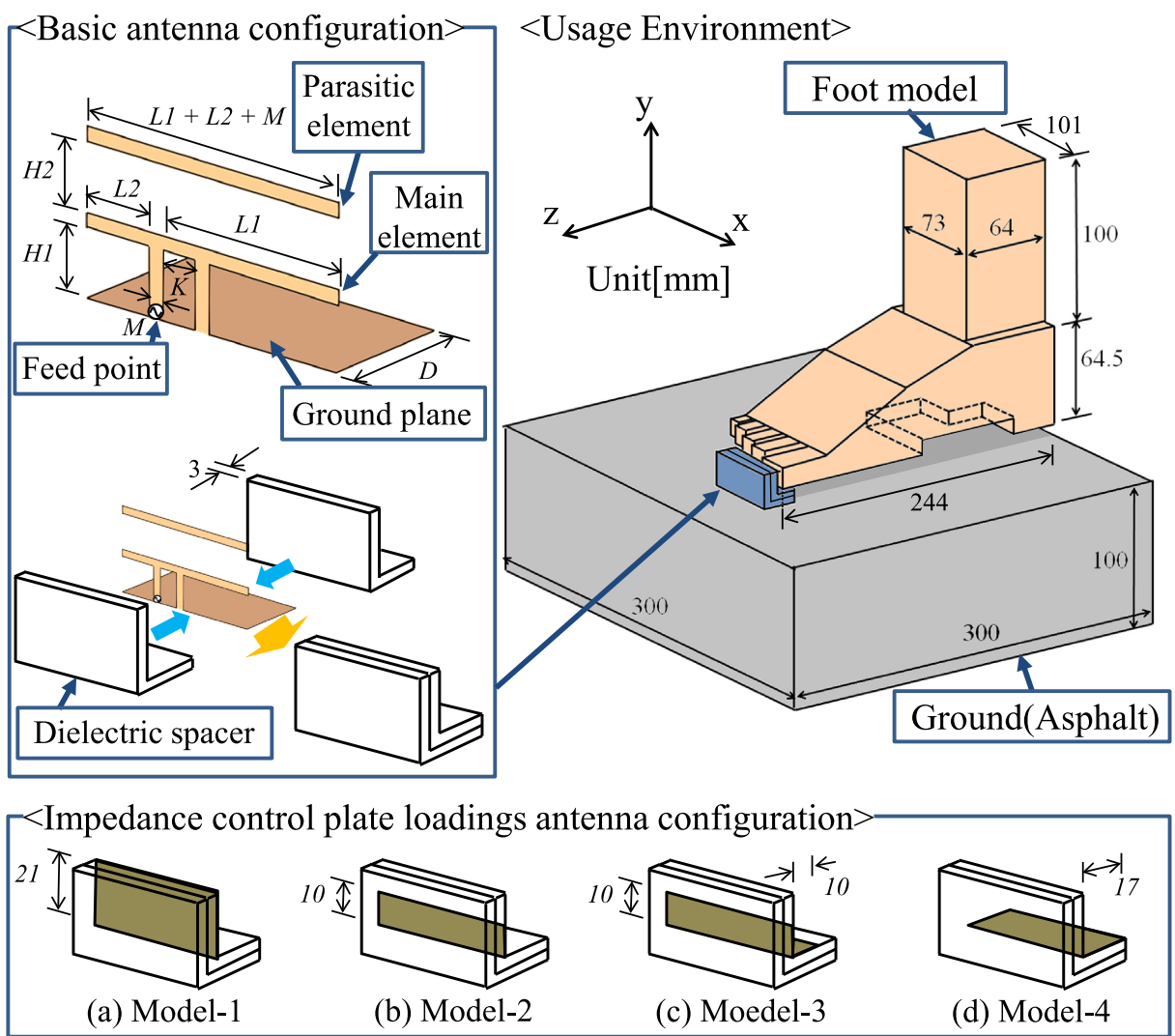

Fig. 1. The structure of proposal antenna and loading situation of the actual antenna.

\section{Simulated results and discussion}

The Finite Difference Time Domain (FDTD) method is used for antenna design and antenna properties estimation [6]. The Fig. 2(a) shows the result of antenna input properties in ideal situation (in free space) and actual use environment. As results in Fig. 2(a), it is confirmed that antenna input properties slightly deteriorate when foot or ground existed. To reduce the influence by the foot and ground, an impedance control plate is mounted on the backside of the dielectric spacer as shown in Fig. 1. Four models were designed for the optimization of impedance control metal plate loading, and the performance of each model was verified. As for the Model-1, antenna elements are totally covered with the impedance control plate. In Model-2, only an antenna main element is covered with impedance control plate, and a parasitic element is not covered. As for Model-3, a part of ground plane is covered with the impedance control plate, in addition to the Model-2. It is Model-4 that only the ground plane is covered with the impedance control plate. The input properties as the function of the frequency with four models are shown in Fig. 2(b). As results, it was confirmed an antenna input property deteriorated by the foot and ground existence be able to cancel with the impedance control plate loadings. 
Consequently, Model-3 shows the best antenna input properties at both of GPS and Wi-Fi frequency bands.

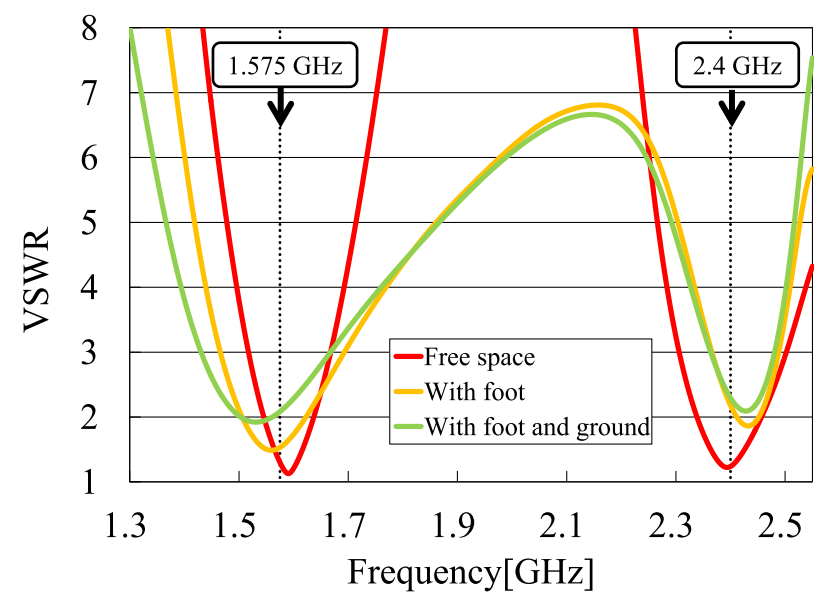

(a) Environmental dependency of VSWR of the antenna without radiation control plate.

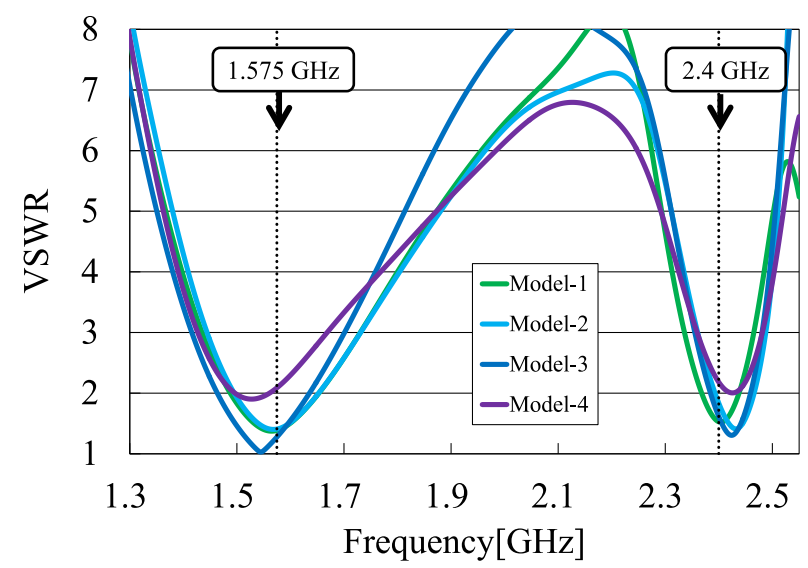

(b) Influence evaluation to the radiation control plate of the antenna's VSWR

Fig. 2. Antenna input property as a function of frequency.

Fig. 3(a) shows analysis results of radiation properties in GPS-band and Wi-Fi band under the situation of Fig. 1. When the foot and ground are adjacent to the antenna, the maximum radiation gain reaches $4.1 \mathrm{dBi}$ at direction of $50^{\circ}$ on xy plane at GPS-band. Also, when the foot and ground are adjacent to the antenna, the maximum radiation gain reaches $6.9 \mathrm{dBi}$ at direction of $0^{\circ}$ on xy plane at Wi-Fiband. The radiation gain in the direction of the zenith is emphasized by the influence of ground. Since the signal of GPS satellite comes from the zenith direction, it is preferable that the radiation gain in the $0^{\circ}$ direction becomes high.

Fig. 3(b) shows analysis results of radiation properties in GPS-band and Wi-Fi band under the impedance control plate loadings situation. The maximum radiation gain of Model-3 that the input characteristic was the best reaches $2.7 \mathrm{dBi}$ at direction of $50^{\circ}$ on xy plane at GPS-band. Moreover, the maximum radiation gain of Model-3 reaches $6.7 \mathrm{dBi}$ at direction of $0^{\circ}$ on xy plane at Wi-Fi band. Accordingly, it was confirmed that the impact to the radiation property is few though the loadings of impedance control plate some decrease the maximum radiation gains at 


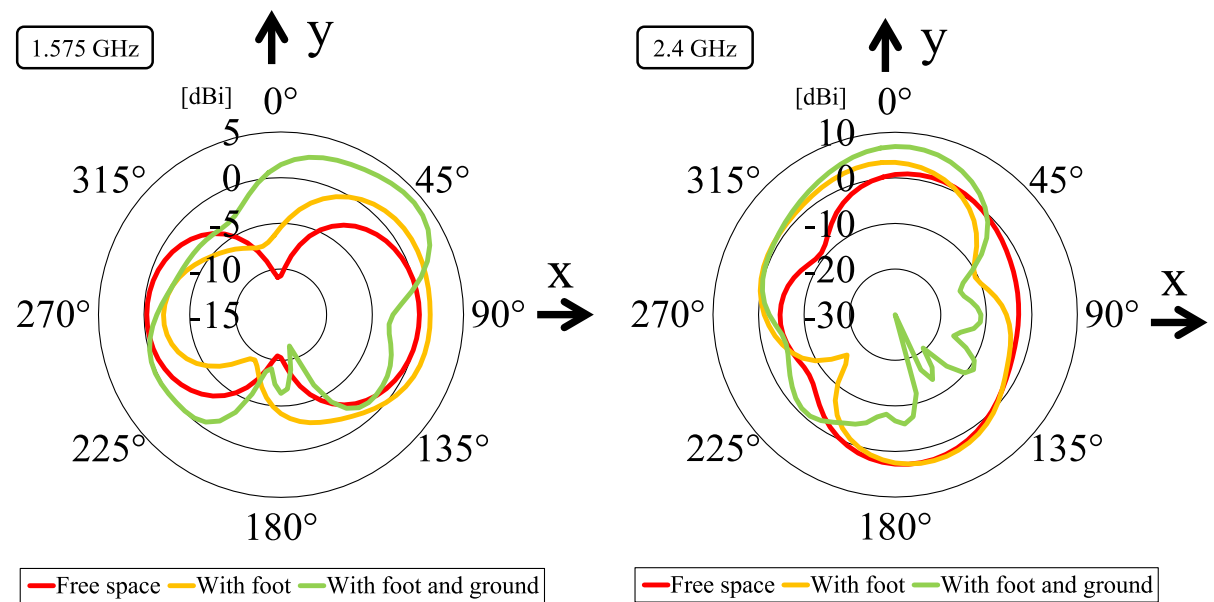

(a) Environmental dependency of radiation of the antenna without radiation control plate.
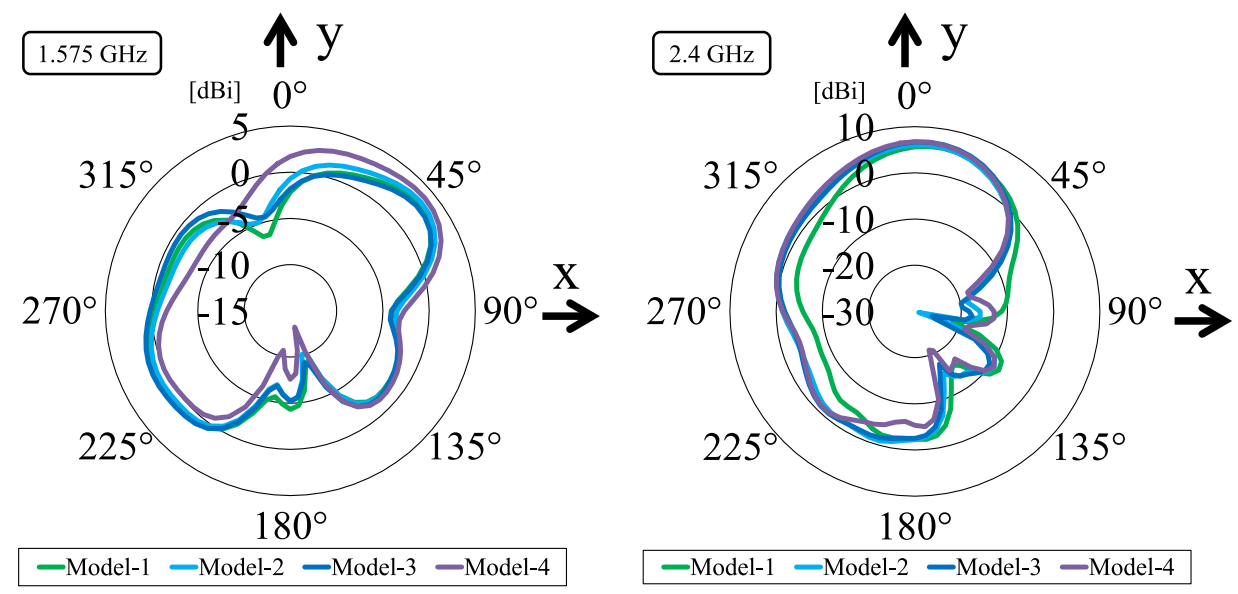

(b) Environmental dependency of radiation of the antenna with radiation control plate.

Fig. 3. Radiation patterns $E \varphi$ xy plane at $1.575 \mathrm{GHz}$ and $2.4 \mathrm{GHz}$.

\section{Conclusion}

This letter proposed the shoes mountable dual-band antenna which can correspond to the GPS signal reception, and the uplink into the wide area network. It was shown to be able to achieve the dual-band antenna for shoes loadings by simple modified inverted-F antenna. However, it was also confirmed that the input property of proposed antenna slightly deteriorated when the foot or ground existed. Consequently, to reduce the influence by the foot and ground, an impedance control plate was mounted on the backside of the antenna. It was confirmed that the impact to the antenna radiation property was comparatively small though the loadings of impedance control plate was effective in the control of the antenna input property. At target frequencies, input properties with final configuration of antenna could satisfy the requirement (VSWR less than 1.5 at $1.575 \mathrm{GHz}$, VSWR less than 1.5 at $2.45 \mathrm{GHz}$ ). Moreover, the maximum radiation gain in GPS-band of the proposal antenna reached $2.7 \mathrm{dBi}$ and also the maximum radiation gain in Wi-Fi-band reached $6.7 \mathrm{dBi}$. The prototype of this antenna will be fabricated in the future, and the demonstration test will also be carried out. 